\section{PTU-158 BOTULINUM TOXIN USE IN OESOPHAGEAL DYSMOTILITY}

${ }^{1}$ MGO Saunders*, ${ }^{2} \mathrm{KL}$ Woolson, ${ }^{3} \mathrm{CR}$ Chimakurthi, 'DK George. 'Gastroenterology, South Devon Healthcare NHS Foundation Trust, Devon; ' Gastroenterology, Royal Cornwall Hospitals NHS Trust, Truro; ${ }^{3}$ Gastroenterology, Plymouth Hospitals NHS Trust, Plymouth, UK

\subsection{6/gutjnl-2014-307263.232}

Introduction Oesophageal dysmotility represents a treatment challenge, with many patients refractory to medical management. There is a lack of evidence for the treatment of oesophageal dysmotility, with only a handful of studies into the use of Botulinum Toxin (Botox) injections, the largest being of 29 patients in 2002. ${ }^{1}$ Our retrospective, multi-centre study assessed the efficacy of Botox injections for oesophageal dysmotility in the South West of England.

Methods The pharmacy databases in three hospitals were interrogated for Botox released to the gastroenterology departments, between January 2009 and December 2013. Then electronic endoscopy databases were utilised to identify those patients treated for oesophageal dysmotility. Patients with achalasia were excluded. Clinical notes were reviewed looking at prior investigations, treatments and presenting symptoms. The numbers of treatments, symptom improvement, duration of response and morbidity or mortality associated with treatment were also assessed.

Results Forty-three patients with oesophageal dysmotility were treated with Botox (mean age 69 years, range 25-95), with a mean of 2.8 treatments per patient (range 1-19). The main presenting symptom was dysphagia $(\mathrm{n}=38)$, either alone or with chest pain, vomiting, reflux or regurgitation. All patients had failed at least one pharmacological treatment, with 11 patients having tried over three different treatments, prior to Botox.

A good treatment response was reported by $56 \%(n=24)$ of patients with their first injection. There was a variable duration of response, from three months to five years, with an average response of 12 months. In 25\% ( $n=6)$ of patients with a good initial response, further treatments were not as effective. There were a variety of injection techniques used, by different endoscopists, with no obvious difference in success rates between the techniques. There were no immediate post-procedure complications. Four patients died within 30 days of Botox injection, all of whom were on an end of life pathway.

Conclusion Botox can be a useful treatment in oesophageal dysmotility; however, careful patient selection is important. Further research is needed into the most effective injection technique and whether there are any patient predictors of response.

\section{REFERENCE}

1 Miller LS, Pullela SV, Parkman HP, Schiano TD, Cassidy MJ, Cohen S, Fisher RS. Treatment of chest pain in patients with noncardiac, nonreflux, nonachalasia spastic esophageal motor disorders using botulinum toxin injection into the gastroesophageal junction. Am J Gastroenterol. 2002;97(7):1640.

Disclosure of Interest None Declared.

\section{PTU-159 VARIABLE UTILITY OF CHROMOGRANIN A ASSAYS IN THE DIAGNOSIS OF GASTRIC CARCINOID TYPE 1}

${ }^{1,2}$ RE Rossi, ${ }^{3} \mathrm{NG}$ Martin, ${ }^{1} \mathrm{~J}$ Garcia-Hernandez, 'D Mandair, ${ }^{1} \mathrm{M}$ Mullan, ${ }^{1} \mathrm{C}$ Toumpanakis, ${ }^{1}$ ME Caplin*. 'Neuroendocrine Tumour Unit, Centre for Gastroenterology, Royal Free Hospital, London, UK, London, UK; ${ }^{2}$ Postgraduate School of Gastroenterology, Universita'degli Studi Di Milano, Milan, Italy; ${ }^{3}$ Department of Clinical Biochemistry, Royal Free Hospital, London, UK

10.1136/gutjnl-2014-307263.233
Introduction Chromogranin A (CgA) is used in the diagnosis and follow-up of patients with neuroendocrine tumours, whilst there is debate over the accuracy of CgA assays in gastric carcinoid type 1 (GC1). Clinical interpretation of $\mathrm{CgA}$ results may be affected by the heterogeneity between available assays. The commercial CgA assay, DAKO (DAKO, Denmark A/S, Glostrup, Denmark) is an ELISA which recognises a $23 \mathrm{kD} C$ terminal fragment of CgA; the Imperial Supra-regional Assay Service radioimmunoassay (SAS Hammersmith Hospital, Imperial College, London) is a competitive radioimmunoassay raised against the whole pancreastatin molecule. Present study is aimed at comparing CgA-DAKO and CgA-SAS to determine their accuracy in the diagnosis of GC1.

Methods Patients with a confirmed diagnosis of GC1 and available plasma CgA measurements according to two different assays (SAS, DAKO) were included and retrospectively reviewed. CgA values were ranked in 4 groups: 1 . normal values, 2 . increase $<2$ upper limit of normal (ULN), 3.increase between 2-5 ULN, 4. increase $>5$ ULN.

Results 26 patients, 17 female and 9 male, mean age 55 years \pm 11.75, were identified. At diagnosis, median CgA-DAKO were significantly higher than median CgA-SAS (81, normal range $<27 \mathrm{IU} / 1$ versus $34.5 \mathrm{pmol} / \mathrm{l}$, normal range $<60 \mathrm{pmol} / \mathrm{l}, \mathrm{T}=35.5$, $\mathrm{p}<0.001)$. When ranking the data, the results confirmed median CgA-DAKO significantly higher than median CgA-SAS: 3 vs. $1, \mathrm{~T}=0, \mathrm{p}<0.001$. Sensitivity was $77 \%$ and $7.7 \%$ for CgA-DAKO and CgA-SAS, respectively.

Conclusion CgA-DAKO shows a better sensitivity than CgA-SAS for the diagnosis of GC1. Accurate diagnostic biomarkers may identify those patients who may benefit from a closer endoscopic follow-up in cases of raised neuroendocrine markers. Further prospective studies are needed highlighting the difference in diagnostic sensitivity between assays.

\section{REFERENCES}

1 Ramachandran $R$, et al. Improved diagnostic accuracy for neuroendocrine neoplasms using two chromogranin A assays. Clin Endocrinol (Oxf). 2012;76:831836

2 Baudin $\mathrm{E}$, et al. Impact of chromogranin-A measurement in the work-up of neuroendocrine tumors. Ann Oncol 2001;12(Suppl 2): S79-S82

3 Stridsberg $\mathrm{M}$, et al. A comparison between three commercial kits for chromogranin A measurements. Journal of Endocrinology 2003:177:337-341

4 Ardill JES. Circulating markers for endocrine tumours of the gastroenteropancreatic tract. Annals of Clinical Biochemistry 2008:45, 539-559

Disclosure of Interest None Declared.

\section{PTU-160 SO YOU REQUESTED COELIAC SEROLOGY; WHAT NEXT?: A SEVEN YEAR REVIEW OF OUTCOME AFTER REQUESTING ANTI-TISSUE TRANSGLUTAMINASE}

${ }^{1} \mathrm{MW}$ Johnson*, ${ }^{2} \mathrm{D}$ Housley, ${ }^{3} \mathrm{D}$ Gordon, ${ }^{1} \mathrm{~K}$ Rostami. ${ }^{1}$ Gastroenterology, Luton and Dunstable FT University Hospital, Luton, UK; ${ }^{2}$ Biochemistry, Luton and Dunstable FT University Hospital, Luton, UK; ${ }^{3}$ General Medicine, Luton and Dunstable FT University Hospital, Luton, UK

\subsection{6/gutjnl-2014-307263.234}

Introduction Coeliac disease is common in the UK with a prevalence of 1 in every 100-200 of the population. Individuals may go undetected for many years, despite presenting numerous times to both primary and secondary care. Some of the delay in diagnosis and missed diagnoses, may reflect that fact that coeliac disease can be asymptomatic or present with very subtle gastrointestinal symptoms. Serological testing is simple and accurate with studies suggesting sensitivity and specificity to be in the order of $95-98 \%$ and $95-97 \%$, respectively. Not surprisingly 
therefore biochemistry laboratories throughout UK receive a substantial number of requests for coeliac serological assessments every year.

Objective We set out to review the total number of requested anti-endomysial antibody (AEAs) and anti-tissue transglutaminase (ATTG) tests between 2007 and 2013 at a busy district general hospital, and reviewed the outcome of having made that request. Methods The results of all the coeliac serology requests made during the study period were reviewed and a retrospective analysis was made of the hospital records to find out the outcome in all those patients with positive results. The laboratory issued a positive result if the ATTG was greater or equal to 4 .

Results During 2007, 810 AEAs were requested, of which 30 $(3.7 \%)$ were positive (10 were weak positive), 736 were negative, 44 were not done. In 2008 ATTG became the primary coeliac serology test and AEAs were only used to review gluten free dietary (GFD) compliance. Between Jan 2008 and Dec 2013 a total of 20,677 ATTGs were requested. This has steadily increase year on year. In 2008 there were 913 requests, 1,389 in $2009,3,060$ in 2010, 4,238 in 2011, 5,584 in 2012 and 6,483 in 2013 . Of these 785 (3.6\%) proved positive, 19891 were negative, 372 samples were deemed insufficient and 605 were rejected by the laboratory as not indicated. Histological confirmation of coeliac disease was made in 222 patients, however a large proportion of positive serology received no further assessment.

Conclusion There is an ever increasing number of requests for coeliac serology, costing our local CCG $£ 21,070$ in 2013. Despite the positive pick up rate being high at $3.6 \%$, a large number of positive results were not pursued any further, with patients failing to have a definitive diagnosis made. It is important to ensure that there are robust mechanisms of chasing up on hospital results, and acting on them appropriately to prevent delayed or missed diagnoses.

Disclosure of Interest None Declared.

\section{PTU-161 ENDOSCOPIC BOUGIE DILATATION IS EFFECTIVE AND SAFE FOR OES OPHAGEAL AND PHARYNGEAL STRICTURES: OUTCOMES OF A LARGE CASE SERIES}

MR Smith*, B Drinkwater, M Widlak, NC Fisher. Gastroenterology, Dudley Group Hospitals NHS Foundation Trust, Dudley, UK

\subsection{6/gutjnl-2014-307263.235}

Introduction Endoscopic bougie dilatation is a traditional technique for managing oesophageal strictures. There are some safety concerns with this technique, but no corroborative evidence of this in controlled or uncontrolled studies to date.

Methods We evaluated the outcomes and safety of endoscopic bougie dilatation at our centre, using the endoscopy database to identify all dilatations done by a single operator. Bougies were the preferred option for all dilatations. All cases from January 2007 to March 2013 were then reviewed by case note analysis.

Results 146 patients were identified, who underwent a total of 346 bougie dilatations. Median age was 67 yrs (range 27-91). Indications for dilatation were: peptic stricture 80 (55\%), malignant stricture $20(14 \%)$, post-surgical stricture $12(8 \%)$, pharyngeal pathology $25(17 \%)$ and other 9 (6\%). Pharyngeal pathology was predominantly post-radiotherapy strictures (64\%) and neurological (20\%).

In cases of peptic stricture, $78 / 80(98 \%)$ had a good symptomatic response to an initial course of dilatation (requiring 1 procedure only in $82 \%$ ). Median end dilatation diameter was
$17 \mathrm{~mm}$ (range 12-18). Recurrence requiring further dilatation occurred in 27 (34\%), after a median of 8 months (range 3-47). In the remainder, median observed remission was 24 months (range 1-63). For pharyngeal pathology patients underwent a median of 2 dilatations (range 1-12). After initial dilatation, 12 (48\%) achieved lasting benefit, 5 (20\%) had no benefit and 8 (32\%) benefited from periodic scheduled dilatations.

Overall median follow up was 22 months (IQR 7- 48). Among the whole case series there were $6(4 \%)$ unscheduled admissions, all self-limiting (dysphagia 2, food bolus 2, stentrelated bleed 1, pain 1). There were no perforations.

Conclusion This large case series supports the role of bougie dilatation as a safe and effective therapy for benign peptic strictures. With careful case selection it also appears a valuable, appropriate and safe option for a range of similar oesophageal and pharyngeal pathologies.

Disclosure of Interest None Declared.

\section{PTU-162 THE EPIDEMIOLOGY, CLINICOPATHOLOGICAL CHARACTERISTICS ND OUTCOMES OF GISTS IN THE NORTH EAST OF ENGLAND OVER A FIVE YEAR PERIOD}

${ }^{1}$ MJ Friel* ${ }^{*}{ }^{2} Y$ Vishwanath, ${ }^{2} \mathrm{H}$ Wescott, ${ }^{3} \mathrm{~N}$ Wadd, ${ }^{1} \mathrm{~A}$ Dhar. ${ }^{1}$ Gastroenterology, County Durham and Darlington NHS Foundation Trust, Co. Durham, UK; ${ }^{2}$ Surgery, James Cook University Hospital, Middlesbrough, UK; ${ }^{3}$ Oncology, James Cook University Hospital, Middlesbrough, UK

\subsection{6/gutjnl-2014-307263.236}

Introduction Gastrointestinal stromal tumours (GISTs) are rare mesenchymal tumours of the gastrointestinal tract. In recent years there is increasing focus on immunohistochemistry biomarkers and targeted Imatinib therapy for treatment, but there is little data from the UK on factors that influence outcome.

Methods We reviewed clinical, pathological, treatment strategies, follow-up and outcome data in all patients with GISTs at our regional multidisciplinary cancer group between Jan 2008 and Dec 2012. Tumour size, mitotic index, other pathological parameters and immunohistochemical stains including CD117 (KIT), CD34, and others were recorded. Tumours were categorised according to the NIH, revised AFIP, and AJCC risk-stratification models. Cox proportional hazard regression was used to determine independent factors associated with survival.

Results 42 patients with GIST were identified. 36(85.7\%) were located in the stomach, $5(11.9 \%)$ in the small intestine, and 1 $(2.4 \%)$ in the oesophagus. Median age was 68 (range 43-91) yrs. $24(57.1 \%)$ were female. Tumour size ranged from 1.0$12.7 \mathrm{~cm}$ (mean $5.5 \mathrm{~cm}$ ). Metastasis was present in $19(45.2 \%)$ at diagnosis, the liver being the most common site in $8(42.1 \%)$. Histology and immunohistochemical analysis was available in 31 (73.8\%). Commonest histological subtype was spindle cell in 17 (53.1\%), epitheloid in 9 (29.0\%) and mixed in 5 (16.1\%). CD117 was positive in 90.6\%, and CD34 in 75.0\%. 54.8\% patients underwent surgical resection with radical surgery in $47.8 \%, 5$ of whom received extensive adjacent organ resection. $47.8 \%, 34.8 \%$ and $52.2 \%$ patients were categorised as high risk according to NIH, AFIP and AJCC (stage III-IV) risk models respectively. Recurrence was confirmed in $5(11.9 \%)$ patients at a median of 597 (range 402-943) days. Of these, 2 were deemed low risk by all three classification systems. Imatinib was given to $14 / 42(33.3 \%)$ patients; as primary therapy in $10(28.3 \%)$ patients (9 palliative and 1 neoadjuvant), and as adjuvant therapy in 4 patients. Cox proportional hazard regression revealed age, tumour site, size, mitotic count, metastases at 